\title{
Automated Analysis of Natural Language Properties for UML Models*
}

\author{
Sascha Konrad - Betty H.C. Cheng ${ }^{\dagger}$ \\ \{konradsa,chengb\}@cse.msu.edu \\ Software Engineering and Network Systems Laboratory \\ Department of Computer Science and Engineering \\ Michigan State University \\ 3115 Engineering Building \\ East Lansing, Michigan 48824 USA
}

\begin{abstract}
It is well known that errors introduced early in the development process are commonly the most expensive to correct. The increasingly popular model-driven architecture (MDA) exacerbates this problem by propagating these errors automatically to design and code. This paper describes a round trip engineering process that supports the specification of a UML model using CASE tools, the analysis of specified natural language properties, and the subsequent model refinement to eliminate errors uncovered during the analysis. This process has been implemented in SPIDER, a tool suite that enables developers to specify and analyze a UML model with respect to behavioral properties specified in terms of natural language.
\end{abstract}

\section{Introduction}

Errors introduced early in the development process are known to have above-average correction costs [14]. To worsen this problem, in the increasingly popular modeldriven architecture (MDA) [22], platform-independent models are transformed to platform-specific models via transformation techniques. As such, these errors are directly propagated to the platform-specific models and may also be propagated to

\footnotetext{
${ }^{*}$ This work has been supported in part by NSF grants EIA-0000433, EIA-0130724, CDA-9700732, CCR-9901017, Department of the Navy, Office of Naval Research under Grant No. N00014-01-1-0744, Eaton Corporation, Siemens Corporate Research, and in cooperation with Siemens Automotive, Detroit Diesel Corporation, and General Dynamics Land Systems.

$\dagger$ Please contact this author for all correspondences.
} 
code, thereby motivating their detection in the platform-independent models. Validating UML models according to metrics and design guidelines can be an effective means to catch structural errors [1] 3], but generally not behavioral modeling errors. Several tools for the behavioral analysis of UML models have been developed, where a user typically specifies properties in terms of formal specification languages. However, these formal specification languages often have a complex syntax and semantics and are, therefore, rarely used in practice. To ease the use of formal specification languages, we have developed a customizable process for specifying properties of formal system models in terms of natural language and formally analyzing these properties using various formal analysis tools [11.

Several other tools exists to support the design and validation of system models. Commercial tools commonly offer validation and/or animation capabilities, such as Rhapsody [7] and Rational XDE [8]. In general, these tools aid in uncovering structural errors, but are not designed for the analysis of behavioral properties of a system model. Other tools have been developed for the formal analysis of system models specified in terms of UML, such as vUML [13], Hugo [25], and Fujaba [18]. However, these tools have still not gained a widespread use in industry. One main reason is the need to use complex specification logics and/or formal analysis tools. Consequently, only users with an advanced knowledge in formal methods are inclined to use these tools for the specification and analysis of their system models.

In this paper, we present three main contributions: First, we developed a process for specifying and analyzing formal properties, where the objective is to make the formal nature transparent to the user. As such, property templates based on specification patterns developed by Dwyer et al. [5] can be specified in natural language and used to analyze the system model. We implemented this process in SPIDER (Specification Pattern Instantiation and Derivation EnviRonment), and we show how SPIDER can be used in combination with a previously developed UML formalization framework, termed Hydra [16], for the analysis of UML models. Second, to facilitate the specification process, we provide support for instantiating the natural language property templates with information that is automatically extracted from the formal system model under consideration. Third, the process is customizable for different domain-specific natural language vocabularies and specification styles, specification pattern systems, and analysis tools.

In this work, we show how our process can be used to specify and analyze natural language properties of UML models. More specifically, our round trip engineering process is configured to read UML 1.4 20] models specified in terms of XMI 1.1 19] and generate the formal specification language Promela for the model checker Spin [6]. Natural language properties are derived using a previously developed grammar [12] that supports the specification patterns by Dwyer et al. [5]. Our grammar enables the natural language representation of these specification patterns. In this paper, the grammar is used to specify linear-time temporal logic (LTL) properties [15], the property description language of the Spin model checker. The grammar

\footnotetext{
${ }^{1}$ CASE tool support for the recently finalized UML $2.0[21$ is still limited.
} 
can be customized according to vocabulary and specification style of a domain. For example, the vocabulary and natural language specification style to capture a causeeffect property for the embedded systems domain may be different from that used for a web service application. As such, the mappings from the structured natural language grammar to the specification patterns should reflect the appropriate intent. In addition, the semantic UML mapping rules of Hydra can be customized and adapted to other domains [16]. In this paper, we use a semantic interpretation considered to be suitable for the embedded systems domain. Our approach does not require the user to know the specific syntax and semantics of the formal specification language used or the details of the analysis procedures of the targeted formal analysis tool. An analysis process can be automatically executed and the analysis results are displayed to the user in a form easy to comprehend.

Overall, we introduce a customizable process that combines the completeness of a pattern system for specifying properties of UML models with the accessibility of a natural language representation, and present a prototype implementation termed SPIDER. To validate our approach, we have applied the process and tools to several examples from industry, including an electronically controlled steering system and an adaptive light control system. The remainder of the paper is organized as follows. Section 2 describes our process and the main components of SPIDER in more detail. Section 3 examines related work. Finally, Section 4 gives concluding remarks and discusses future work.

\section{Specification and Analysis}

In this section, we introduce our specification and analysis process, and we also overview major SPIDER elements. Figure 1 contains a UML activity diagram overviewing our process, where the first two steps of our process are initialization steps and can be performed in any order. The shaded swimlane indicates this portion of the process performed by an administrator for domain customization purposes.

\section{(1) Configuring the Process and Deriving a Property}

In the first initialization step, a specification pattern system has to be created. A specification pattern system is a collection of properties, specified in terms of one or more formal specification languages, with an accompanying natural language grammar, containing natural language representations of all properties. In SPIDER, the Pattern System Manager (shaded portion of Figure 1) is used to create and associate formal properties to their natural language representations, or a previously created pattern system can be loaded. For this paper, the specification pattern system consists of formal properties from the specification patterns by Dwyer et al. 5 and a corresponding natural language grammar [12]. The specification pattern system contains several patterns applicable to software properties specified in different formalisms, such as LTL [15], computational tree logic (CTL) [4], graphical interval logic (GIL) [24], and quantified regular expressions (QRE) [23]. Specification patterns are catego- 


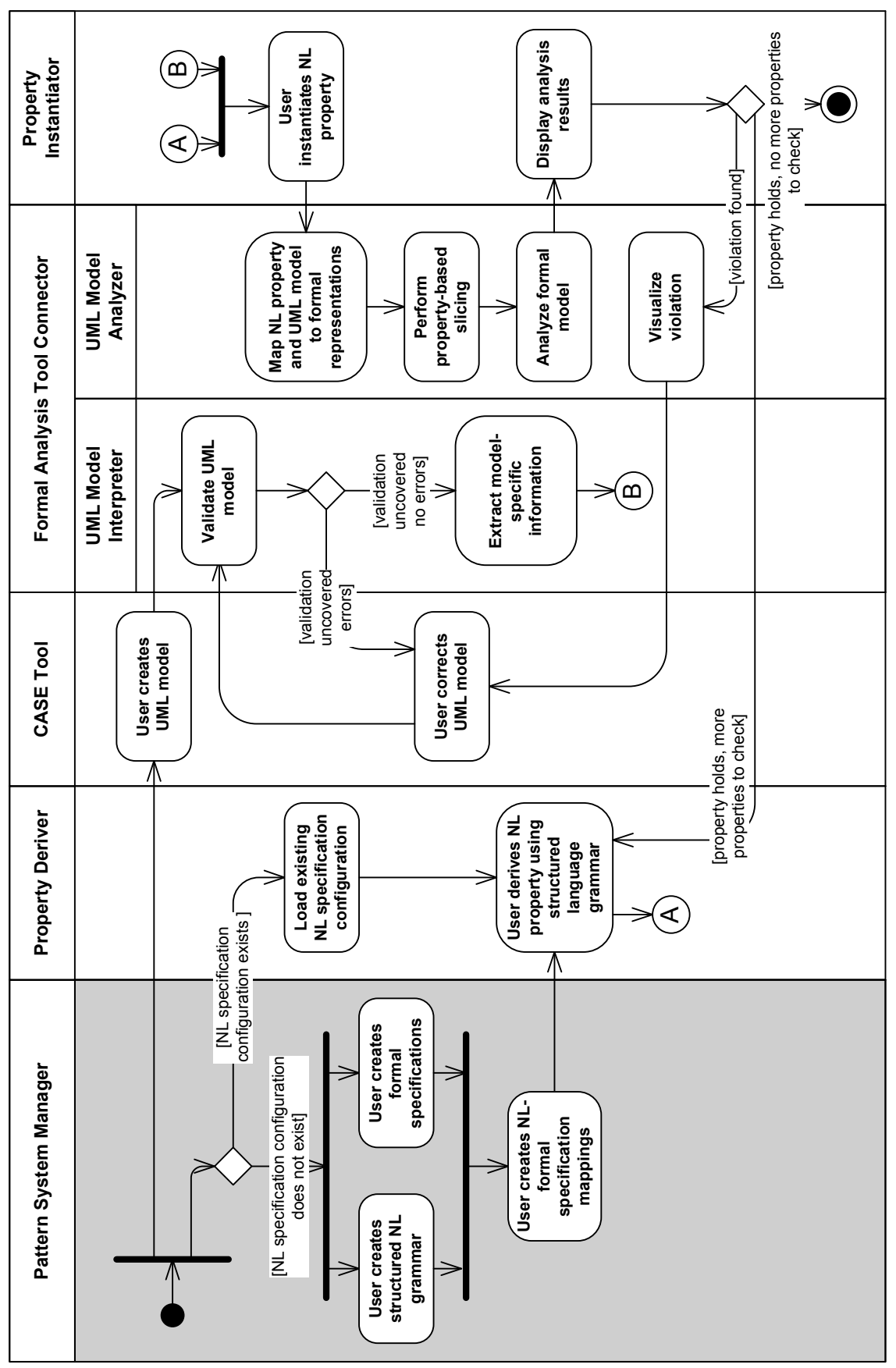

Figure 1: UML activity diagram overviewing our specification and analysis process 
rized into two major groups: occurrence patterns and order patterns. Occurrence patterns are concerned with the occurrence of single states/events during the execution of a program, such as existence and absence of certain states/events. Order patterns, on the other hand, are concerned with the relative order of multiple occurrences of states/events during the execution of a program, such as precedence or response relations between states/events. The specification patterns have been found sufficient to specify most commonly occurring properties 5 . However, while the pattern system is largely reusable, the structured natural language grammar may have to be adapted to accommodate the specification style of a specific domain.

The Pattern System Manager is intended to be used by domain experts and formal methods experts as an administrative tool that configures SPIDER according to a specification pattern system. It aids in the construction and management of specification pattern systems with their associated structured natural language grammars. Structured natural language grammars are captured in Extended Backus-Naur Form (EBNF) and internally translated into a BNF representation. For grammar rules containing choices, additional descriptors are included. These descriptors comprise two parts: an abbreviated name of the choice and a textual explanation of each choice. This information is used in the derivation process to provide guidance and feedback to the user when making a choice in the derivation process. The Pattern System Manager is also used by the formal methods experts to create the mappings between the sentences generated from the natural language grammar and elements from the specification pattern system.

After the process has been instantiated with a natural language grammar and mappings to a specification pattern system, the property to be analyzed is derived. In SPIDER, the Property Deriver is used to guide the user in a stepwise fashion in constructing a structured language sentence capturing the property. Non-terminals are highlighted in the sentence that is being derived and the user resolves these non-terminals with applicable production rules. The Property Deriver assists the user in making specification choices by offering descriptive information about the consequences of each choice. Each time the user highlights a particular choice, the Property Deriver highlights corresponding descriptors. In addition, the Property Deriver gives a preview of selecting a particular choice for the sentence being derived. After the sentence is derived, the first step ends in the connector A in Figure 1

\section{(2) Creating the UML Model}

In the second initialization step, a UML model is created using a CASE tool. To include the model in our process instantiation, the model is exchanged with SPIDER using XMI [19]. Figure 2 shows an example UML class Class1 with an associated state diagram capturing the behavior of the class. Initially, the model is validated by Hydra using static analysis techniques [2]. The model validation encompasses several checks for intra- and inter-diagram validity, such as checks for well-formedness of names and expressions, missing initial states, states without incoming or outgoing transitions, and undeclared variables, operations, or types. If errors are found during the validation that prevent a formally specified model to be generated from the UML 
diagrams, then the user is prompted to correct these errors before proceeding. After the model passes the validation checks, the UML Model Interpreter automatically extracts information from the UML model. For example, for the UML diagram in Figure 2 , the UML Model Interpreter extracts the following information for class Class1:

Variable names: timer 1

Signal names: reset

State names: Wait, Process

The UML Model Interpreter is part of the Formal Analysis Tool Connector that is used to connect SPIDER with UML tools and the Spin model checker. In general, the tool connector enables SPIDER to extract information from a system model, create formal specifications of properties understood by the targeted formal analysis tool, execute the verification of a property, and analyze the output generated by a verification run of the formal analysis tool. SPIDER offers the ability to plug in additional Formal Analysis Tool Connector components. Therefore, SPIDER is extensible to numerous analysis tools beyond the ones explicitly mentioned in this paper. After the information has been extracted, the second step ends in the connector B in Figure 1

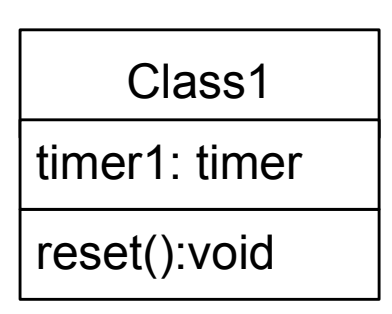

(a) Class Diagram

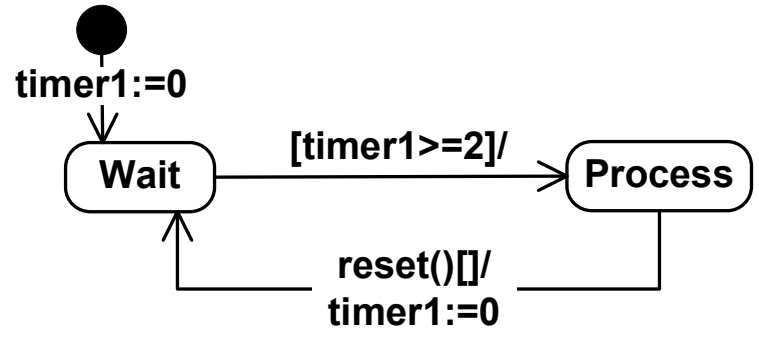

(b) State Diagram

Figure 2: Example UML models

\section{(3) Instantiating the Property}

After the previous two steps are completed in connectors A and B, the information extracted by the UML Model Interpreter is then used by the Property Instantiator to instantiate the structured language sentence with boolean propositions containing model-specific elements. In addition to specifying boolean expressions on variable values of UML classes, two other predicates are supported in the boolean propositions: (1) A call (...) predicate to specify that an operation of a class is called and (2) an enter (...) predicate to specify that a class enters a specific state.

\section{(4) Analyzing the Property}

After the instantiation is complete, the model can be analyzed for adherence to the specified property. In SPIDER, the UML Model Analyzer, which is also part of the Formal Analysis Tool Connector, maps the instantiated natural language sentence to 
the corresponding specification pattern instances, namely LTL formulae [15] for the Spin model checker [6. In order to reduce the cost of model checking, we perform an automated abstraction of the formal model before executing the analysis. The $U M L$ Model Analyzer performs a property-based slicing on the formal system model, where it invokes the slicing algorithm provided by Spin 2 and removes constructs identified as redundant. After the slicing is complete, SPIDER invokes the Spin model checker and performs the analysis.

\section{(5) Displaying Analysis Results}

After the analysis has completed, the UML Model Analyzer provides analysis results back to the Property Instantiator, which are then visually presented to the user using a traffic light icon. Red indicates that the property was violated and a counter example is returned; Green indicates that the property holds for the selected model; and Yellow indicates that problems occurred during the analysis process that prohibited the successful verification of the property. Example problems include exceeding the available system memory for storing the states of the model during an exhaustive state space exploration. If a violation of a property is found, then the user can visualize the execution that lead to the violation, correct the model, and repeat the analysis. Finally, when the property holds on the selected model, the user can analyze additional properties or exit from the tool and the analysis process.

\section{Related Work}

Due to space constraints, we only briefly overview related work. For a more detailed discussion of related work, please refer to [2,11,16].

Several other projects have investigated how to make specification patterns more accessible via more informal representations. Smith et al. developed Propel [26], where they extended the specification patterns by Dwyer et al. [5] to address important and subtle aspects about a property, such as what happens in a cause-effect relation if the cause recurs before the effect has occurred. These extended specification patterns are specified in terms of finite-state automata instead of temporal logic formulae and natural language templates help a specifier to precisely capture a property in natural language. In contrast to our approach, they focus on capturing subtle properties of individual specification patterns, rather than applying the specification patterns to the analysis of UML models. Mondragon et al. developed a tool called Prospec [17 for the specification of properties based on Dwyer et al.'s specification patterns. The tool offers assistance in the specification process and extends the specification pattern system by Dwyer et al. with compositional patterns. Differing from our tool suite, they do not include support for natural language representations.

\footnotetext{
${ }^{2}$ The slicing algorithm of Spin is sound and complete with respect to any property specifiable in terms of LTL 6
} 


\section{Conclusions}

We have presented a configurable process for UML model analysis implemented in the SPIDER toolkit. We expect several benefits to be gained from using SPIDER. First, users less experienced in the specification of formal properties are able to create formally-analyzable natural language representations of properties for their UML models. Feedback from industrial collaborators has indicated that this specification style is preferred over formal specification languages. Second, SPIDER is extensible to the use of several formal analysis tools by offering the ability to plug in additional Formal Analysis Tool Connector components. Therefore, a wide variety of formal analysis tools can be used to analyze the behavioral properties. Currently, SPIDER supports the Spin model checker [6] for UML models and support for additional formal analysis tools is being developed.

Third, SPIDER provides a single environment for specification construction and analysis. The tool suite enables a user to automatically analyze a system model and visualize the analysis results. Currently, our tool is targeted at the novice specifier, as evidenced by the step-by-step guidance during the derivation process and making the formal specification language transparent to the user. We acknowledge that the stepwise, specification-facilitating features, while helpful for the novice user, might be too constraining for users with advanced knowledge in formal specification and analysis. This problem is commonly encountered in syntax-directed editing approaches [9] and we are investigating techniques to mitigate these problems, such as the use of multiple views and different levels of assistance for the derivation and instantiation tasks.

Other directions of future work includes addressing how to incorporate previously developed real-time extensions to our formalization framework [10] and specification patterns [12]. The main issue that needs to be investigated is how to use standard CASE tools to include timers and time invariants into UML models and how to output this information to XMI. This work will also investigate how to best visualize the analysis results. Finally, we also work with industrial collaborators to obtain feedback on the usability of SPIDER.

\section{References}

[1] B. Berenbach. The evaluation of large, complex UML analysis and design models. In Proceeding of the 26th International Conference on Software Engineering (ICSE'04), pages 232-241. IEEE Computer Society, 2004.

[2] L. A. Campbell, B. H. C. Cheng, W. E. McUmber, and R. E. K. Stirewalt. Automatically detecting and visualizing errors in UML diagrams. Requirements Engineering Journal, 7(4):246-287, December 2002.

[3] B. H. C. Cheng, R. Stephenson, and B. Berenbach. Lessons learned from metrics-based automated analysis of industrial UML models (an experience report). In Proceedings of the ACM/IEEE 8th International Conference on Model Driven Engineering Languages and Systems, Montego Bay, Jamaica, October 2005. (Accepted to appear). 
[4] E. M. Clarke, E. A. Emerson, and A. P. Sistla. Automatic verification of finite-state concurrent systems using temporal logic specifications. ACM Transactions on Programming Languages and Systems, (2):244-263, April 1986.

[5] M. B. Dwyer, G. S. Avrunin, and J. C. Corbett. Patterns in property specifications for finite-state verification. In Proceedings of the 21st International Conference on Software Engineering, pages 411-420. IEEE Computer Society Press, 1999.

[6] G. Holzmann. The Spin Model Checker, Primer and Reference Manual. Addison-Wesley, Reading, Massachusetts, 2004.

[7] I-logix. Rhapsody, July 2005. http://www.ilogix.com/rhapsody/ rhapsody.cfm

[8] IBM. Rational Rose XDE Developer, July 2005. http://www-306.ibm.com/ software/awdtools/developer/rosexde/

[9] A. A. Khwaja and J. E. Urban. Syntax-directed editing environments: Issues and features. In SAC '93: Proceedings of the 1993 ACM/SIGAPP Symposium on Applied Computing, pages 230-237. ACM Press, 1993.

[10] S. Konrad, L. A. Campbell, and B. H. C. Cheng. Automated analysis of timing information in UML diagrams. In Proceedings of the Nineteenth IEEE International Conference on Automated Software Engineering (ASE04), pages 350-353, Linz, Austria, September 2004. (Poster summary).

[11] S. Konrad and B. H. C. Cheng. Facilitating the construction of specification patternbased properties. In Proceedings of the IEEE International Requirements Engineering Conference (RE05), Paris, France, August 2005.

[12] S. Konrad and B. H. C. Cheng. Real-time specification patterns. In Proceedings of the International Conference on Software Engineering (ICSE05), St Louis, MO, USA, May 2005.

[13] J. Lilius and I. P. Paltor. vUML: A tool for verifying UML models. In Proceedings of the 14th IEEE International Conference on Automated Software Engineering, page 255, Washington, DC, USA, 1999. IEEE Computer Society.

[14] R. R. Lutz. Targeting safety-related errors during software requirements analysis. In SIGSOFT'93 Symposium on the Foundations of Software Engineering, 1993.

[15] Z. Manna and A. Pnueli. The temporal logic of reactive and concurrent systems. SpringerVerlag New York, Inc., 1992.

[16] W. E. McUmber and B. H. C. Cheng. A general framework for formalizing UML with formal languages. In Proceedings of the IEEE International Conference on Software Engineering (ICSE01), Toronto, Canada, May 2001.

[17] O. Mondragon and A. Q. Gates. Supporting elicitation and specification of software properties through patterns and composite propositions. International Journal on Software Engineering and Knowledge Engineering, 14(1):21-41, February 2004.

[18] U. Nickel, J. Niere, and A. Zündorf. The FUJABA environment. In Proceedings of the 22nd International Conference on Software Engineering, pages 742-745, New York, NY, USA, 2000. ACM Press.

[19] Object Management Group. OMG-XML metadata interchange (XMI) specification, v1.1, 2000. http://www.omg.org/cgi-bin/doc?formal/00-11-02 
[20] Object Management Group. UML Specifications, Version 1.4, 2002. http://wwW. omg.org/cgi-bin/doc?formal/04-07-02

[21] Object Management Group. UML 2.0 Superstructure Specification, 2004. http:/7 www.omg.org/cgi-bin/doc?ptc/2004-10-02

[22] Object Management Group. Model driven architecture. http://www.omg.org/ mda/ 2005.

[23] K. M. Olender and L. J. Osterweil. Cecil: A sequencing constraint language for automatic static analysis generation. IEEE Transactions on Software Engineering, 16(3):268-280, 1990.

[24] Y. S. Ramakrishna, P. M. Melliar-Smith, L. E. Moser, L. K. Dillon, and G. Kutty. Interval logics and their decision procedures: Part I + II. Theoretical Computer Science, 166;170(1-2):1-47;1-46, 1996.

[25] T. Schäfer, A. Knapp, and S. Merz. Model checking UML state machines and collaborations. Electronic Notes in Theoretical Computer Science, 55(3):13 pages, 2001.

[26] R. L. Smith, G. S. Avrunin, L. A. Clarke, and L. J. Osterweil. Propel: An approach supporting property elucidation. In Proceedings of the 24th International Conference on Software Engineering, pages 11-21. ACM Press, 2002. 